\title{
Analyse probabiliste de la stabilité de deux remblais édifiés sur versant instable
}

J.-P. MAGNAN* A. ABDUL BAKI**

\section{P. POUGET**}

* Laboratoire central des Ponts et Chaussées 58, bd Lefèbvre,

75732 Paris Cedex 15

* Laboratoire régional des Ponts et chaussées 8-10, rue Bernard-Palissy BP 11

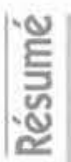

L'article présente l'analyse probabiliste de la stabilité de deux remblais édifiés sur le site expérimental de Sallèdes (Puy-de-Dôme). Cette analyse consiste à évaluer la probabilité de rupture de ces remblais sur la base de la méthode des blocs (découpage du massif susceptible de glisser en blocs rigides) et d'une procédure d'intégration numérique (développement en série de Taylor de la fonction donnant le coefficient de sécurité F).

Les variables aléatoires prises en compte sont les paramètres de cisaillement effectifs des différentes couches rencontrées. La loi de distribution normale a été choisie pour représenter la répartition des valeurs de la cohésion et de l'angle de frottement interne, d'une part, et du coefficient de sécurité, d'autre part.

Les résultats de cette première approche sont comparables avec ceux obtenus dans un calcul déterministe et incitent à poursuivre des recherches dans cette voíe.

\section{Probabilistic analysis of the stability of two embankments built on an unstable slope}

The paper presents the probabilistic analysis of the stability of two embankments built at the Salledes (Puy-de-Dôme, France) experimental site. The analysis consists in the assessment of the probability of failure of these embankments, using the wedge analysis method (which assumes that the sliding mass of soil can be divided into a set of rigid blocks) and of a numerical solving scheme (development of the factor of safety function into a Taylor's series).

The random variables taken into account are the effective shear strength parameters of each soil layer. Normal distributions are assumed for the effective cohesion intercept and the effective angle of internal friction, as well as for the factor of safety. The results of this approximative analysis are similar to those obtained from a deterministic approach; further developments might be of interest. 


\section{Introduction}

Une dizaine de méthodes de calcul probabiliste du comportement d'ouvrages construits sur versants ont été publiées depuis une vingtaine d'années. Ces méthodes restent pour l'instant des produits de recherche sans applications pratiques dans les bureaux d'études. Cette situation est certainement due à l'insuffisance des méthodes testées jusqu'à présent. En effet, il résulte des articles disponibles dans la littérature que l'utilisation des méthodes de calcul probabiliste conduirait à une augmentation parfois sensible mais toujours coûteuse des coefficients de sécurité habituellement admis. Ce caractère pessimiste n'est pourtant pas inévitable: on peut raisonnablement penser que l'amélioration de la description des variations naturelles des propriétés des sols, l'amélioration des méthodes de calcul probabiliste et l'amélioration de la description des mécanismes de rupture devraient conduire à des méthodes de calcul utilisables pour les projets.

Pour cette raison, un travail a été entrepris en 1989 au Laboratoire Régional des Ponts et Chaussées de Clermont-Ferrand visant à utiliser une démarche probabiliste d'intégration numérique sur une méthode simple d'analyse de stabilité consistant à découper le massif en mouvement en différents blocs. Cette technique d'analyse a été appliquée au versant naturel instable du site expérimental de Sallèdes, où deux remblais ont été édifiés et un grand nombre de données sont disponibles.

\section{2}

\section{Le cas traité: le site expérimental de Sallèdes}

Le site expérimental de «versant naturel instable» des Laboratoires des Ponts et Chaussées à Sallèdes (Puy-de-Dôme) a déjà fait l'objet de diverses publications (Morin, 1979; Blondeau et al., 1983; Cartier et Pouget, 1988). Il se caractérise par des formations sédimentaires datant de l'Oligocène supérieur, composées de marnes remaniées ou en place, avec la proximité immédiate d'un dôme volcanique qui est entouré d'éboulis et de colluvions.

On rencontre ainsi sur les six mètres supérieurs des formations essentiellement argileuses qui constituent deux couches (colluvions, marnes remaniées) reposant sur le substratum marno-calcaire. Ces formations meubles glissent lentement au contact avec le substratum. La pente du terrain naturel est de l'ordre de 7 degrés.

Deux remblais ont été édifiés sur le site:

- le premier remblai (remblai A) a été construit en 1978 à des cadences normales de terrassement jusqu'à la rupture;

- le deuxième remblai (remblai B) a été construit en 1980, en deux phases à une hauteur inférieure à celle du remblai A. Le comportement de cet ouvrage est suivi depuis sa réalisation.

L'analyse probabiliste de la stabilité de ces deux remblais est traitée dans cet article.

\section{Analyse de stabilité par la méthode des blocs}

La méthode des blocs repose sur le découpage de la masse de sol susceptible de glisser en blocs rigides dont on analyse l'équilibre sous l'action des forces volumiques (pesanteur), et des efforts de contact entre blocs. Nous avons pris en compte des blocs à interfaces verticaux.

Un programme de calcul a été créé sur la base d’une configuration classique dans le domaine des pentes, qui a conduit à prendre en compte les hypothèses suivantes (Fig. 1):

- le remblai est composé de matériaux homogènes; - la surface supérieure du remblai est horizontale; - le terrain naturel a une pente régulière tout le long du versant;

- les couches de sol sont stratifiées suivant des lignes parallèles au terrain naturel;

- le massif de sol est divisé en quatre blocs:

- EFGE représente le bloc numéro 1,

- GFHIG représente le bloc numéro 2,

- IHKJDCI représente le bloc numéro 3,

- JKLJ représente le bloc numéro 4;

- les points I (commun aux blocs 2 et 3 ) et $G$ (commun au blocs 1 et 2) peuvent se déplacer entre les deux points $A$ et $D$;

- le point $\mathrm{J}$ commun aux blocs 3 et 4 peut se déplacer en partant du point $D$ vers l'aval du remblai;

- les segments GF, IH et JK sont des segments verticaux:

- la pente du segment EF est fixée dès le départ à:

$$
\Omega+\alpha=\frac{\pi}{4}+\frac{\varphi^{\prime}}{2}
$$

$\varphi^{\prime}$ étant l'angle de frottement interne du matériau constituant le remblai;

- les pentes des segments $\mathrm{FH}$ et $\mathrm{KL}$ ainsi que la position des points $\mathrm{H}$ et $\mathrm{K}$ peuvent varier indépendamment, comme nous allons le voir plus loin;

- les forces d'interaction entre blocs (sur les frontières FG, IH et JK) sont supposées horizontales.

La méthode des blocs conduit à calculer les forces appliquées par les blocs 2 et 4 sur le bloc 3 et à déduire de l'équilibre des forces sur ce dernier bloc la valeur du coefficient de sécurité correspondant à la stabilité du massif de sol considéré.

Le coefficient de sécurité F a été pris constant le long de la surface considérée. Ce coefficient est introduit dans la formule de Coulomb de la manière suivante:

$$
T=\frac{1}{F}\left(c^{\prime} 1+N^{\prime} \operatorname{tg} \varphi^{\prime}\right)
$$

où T est la force s'opposant au glissement sur la surface de contact des blocs avec le sol immobile, $N^{\prime}$ est la résultante des forces normales à cette surface, l est la longueur du bloc et $c^{\prime}$ et $\varphi^{\prime}$ sont les propriétés de l'interface.

La détermination de $\mathrm{F}$ résulte du calcul du rapport des forces résistantes aux forces motrices, qui est supposé égal à 1. 

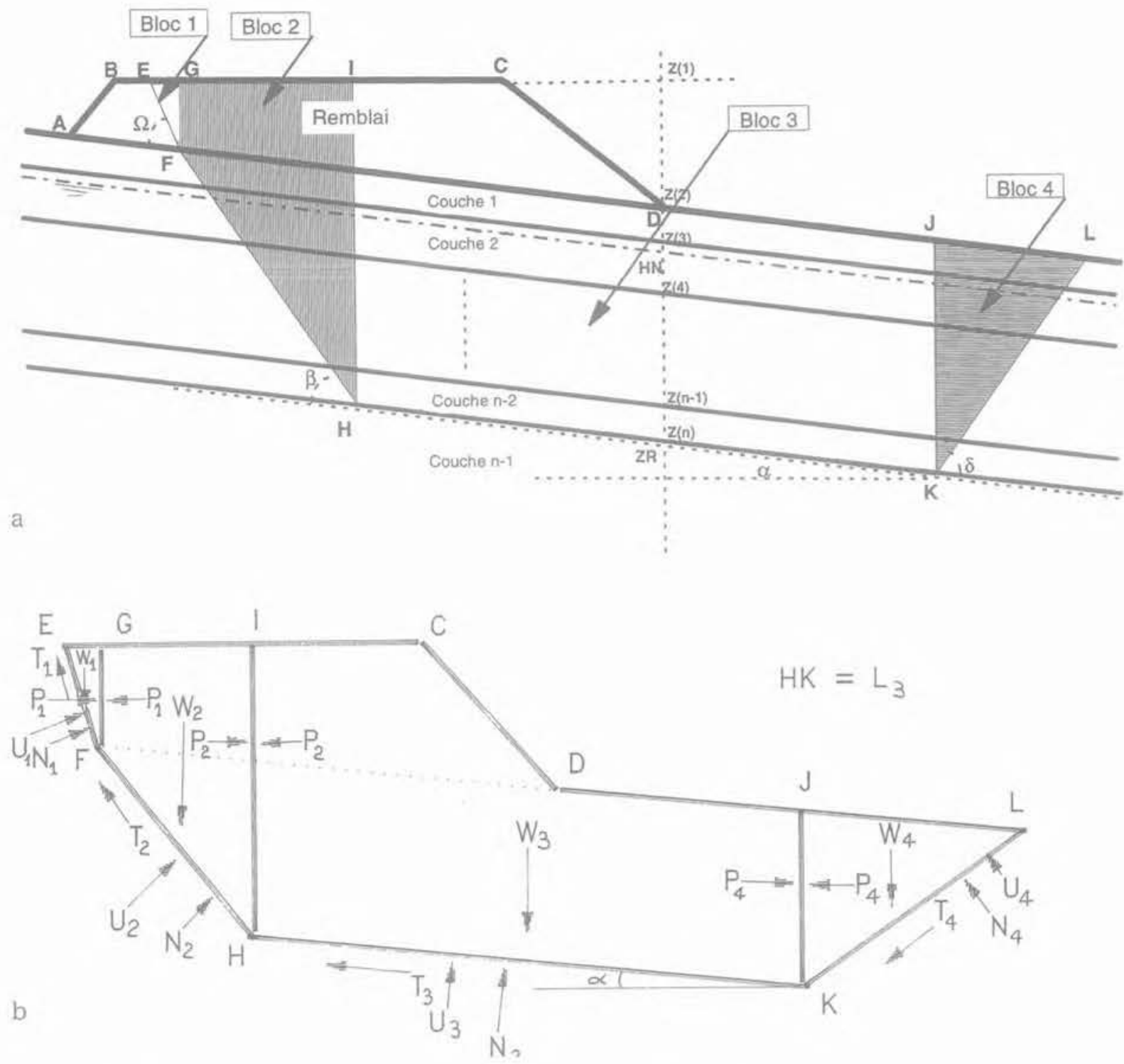

FIG.1 Schématisation de la pente et du remblai pour le calcul.

Design scheme of the slope and the embankment. a. Description du sol, du remblai et du plan de glissement.

a. Description of the soil conditions, the embankment and the failure surface.

b. Forces utilisées dans le calcul d'équilibre, b. Forces used in the stability analysis.

Le calcul des forces $\mathrm{P}_{2}$ et $\mathrm{P}_{4}$ exercées respectivement par les blocs 2 et 4 sur le bloc 3 conduit à diviser les blocs 2 et 4 en autant de blocs que de couches constituant le massif de sol considéré. Ce calcul est effectué sur la base des hypothèses de Bishop.

En écrivant l'équilibre des forces au niveau du bloc 3, le coefficient de sécurité $\mathrm{F}$ est obtenu par la résolution de l'équation suivante:

$$
\begin{aligned}
& W_{3} \cdot \operatorname{tg} \varphi_{n}^{\prime}+P_{4}\left(F+\operatorname{tg} \varphi_{n}^{\prime} \operatorname{tg} \alpha\right)-P_{2} \operatorname{tg} \varphi_{n}^{\prime} \operatorname{tg} \alpha+ \\
& c^{\prime}{ }_{n} L_{3} / \cos \alpha-u_{3}\left(\operatorname{tg} \varphi_{n}^{\prime} / \cos \alpha\right)=F P_{2}+F \cdot W_{3}
\end{aligned}
$$

La racine de cette équation est obtenue par l'application de la méthode de la tangente. Le plan de glissement le plus défavorable est obtenu à partir d'un balayage des points $\mathrm{F}$ et $\mathrm{L}$ d'une part, et des points $\mathrm{H}$ et K d'autre part (Fig. 1)

\section{4}

\section{Technique d'analyse probabiliste utilisée}

Dans une analyse probabiliste, on considère que le résultat du calcul nécessaire à l'étude de la stabilité est une variable aléatoire fonction de plusieurs variables aléatoires caractérisant le sol (Magnan, 1982) :

$$
Y=f(X)
$$

Diverses méthodes peuvent être utilisées pour déterminer de façon approchée la densité de probabilité $\mathrm{g}(\mathrm{y})$ à partir des lois de distribution des paramètres $X$ du modèle de calcul (Rosenblueth, Monte-Carlo, intégration numérique...). La méthode d'approximation par intégration numérique a été utilisée: elle nécessite un développement en série de Taylor de la fonction Y. 
Un développement au second ordre est utilisé ici.

$$
\begin{aligned}
& Y=f\left(X_{i}\right)=f\left(m_{x i}\right)+\sum_{i}^{n} \frac{\partial f\left(x_{i}\right)}{\partial x_{i}} \mid m_{x i}\left(X_{i}-m_{x i}\right)+ \\
& \frac{1}{2} \sum_{i}^{n} \frac{\partial^{2} f\left(x_{i}\right)}{\partial x_{i}^{2}} \cdot m_{x i}\left(X_{i}-m_{x i}\right)^{2}
\end{aligned}
$$

$\mathrm{m}_{\mathrm{x} 1}$ étant la valeur moyenne de $\mathrm{X}$.

L'espérance mathématique de Y s'écrit:

$$
\begin{aligned}
& E[Y]=f\left(m_{x_{i}}\right)+\left.\frac{1}{2} \sum_{i=1}^{n} \frac{\partial^{2} f\left(x_{i}\right)}{\partial x_{i}^{2}}\right|_{m_{x i}} ^{\sigma_{x i}^{2}}+ \\
& \left.\sum_{i=i}^{n} \frac{\partial^{2} f\left(x_{i}\right)}{\partial x_{i} \partial x_{j}}\right|_{m_{x i}} ^{\sigma_{x i v}}
\end{aligned}
$$

Lorsque l'expression de $\mathrm{Y}=\mathrm{f}(\mathrm{X})$ n'est pas connue sous forme analytique, mais peut être déterminée sous forme numérique ou expérimentale, le calcul des dérivées partielles précédentes est pratiquement impossible mais les moments de Y peuvent cependant être déterminés de façon précise par intégration numérique (Lumb, 1974).

La méthode repose sur l'utilisation de la technique d'intégration numérique développée par Evans (1967. 1972), qui peut être présentée de la façon suivante:

$$
\begin{aligned}
& E[Y]=C \cdot f\left(m_{N_{1}}\right)+\frac{1}{2} \sum_{i}^{n} N_{i}\left[\frac{f\left(a_{i}, \sigma_{x i}\right)}{a_{i}}\right]+
\end{aligned}
$$

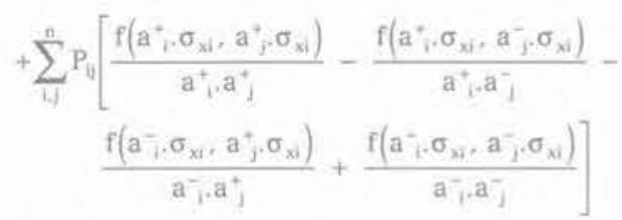

avec les notations suivantes:

$\mathrm{C}, \mathrm{N}_{1}, \mathrm{P}_{1 \mid}, \mathrm{a}^{+}, \mathrm{a}_{\mathrm{p}}^{-}$, sont des constantes,

$\mathrm{f}\left(\mathrm{a}^{+}, \sigma_{\mathrm{xi}}\right)$ est la valeur de $\mathrm{f}\left(\mathrm{X}_{\mathrm{i}}\right)$ au point $\left(\mathrm{m}_{\mathrm{x},}, \ldots\right.$, $\left.\left(\mathrm{m}_{\mathrm{xi}}+\mathrm{a}^{+}, \sigma_{\mathrm{xi}}\right), \ldots, \mathrm{m}_{\mathrm{xn}}\right)$

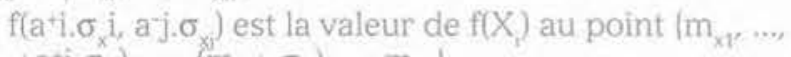
$\left.\left(m_{x i}+a+i . \sigma_{x i}\right), \ldots . .\left(m_{x i}+\sigma_{x}\right), \ldots, m_{x n}\right)$.

La variance de $Y$ est donnée par:

$$
V[Y]=E\left[\left(f(X)-\left.E(f(X))\right|^{2}\right]\right.
$$

Dans le cas où toutes les variables $\mathrm{X}$ ont une distribution normale, on trouve:

$$
\begin{gathered}
a_{i}^{+}=a_{i}^{-}=\sqrt{3} \\
C=1+n(n-7) / 18 \\
N_{i}=-(n-4) /(6 \sqrt{3)} \\
P_{i j}=1 / 12
\end{gathered}
$$

\section{5}

\section{Application au site de Sallèdes}

\section{1}

\section{Choix des variables aléatoires et de leur loi de distribution}

L'analyse statistique de la variabilité des propriétés des sols réalisée sur le site de Salèdes (Abdul Baki, 1992) montre un faible coefficient de variation du poids volumique au niveau de chacun des sondages carottés ( $C \gamma$ ne dépasse pas 0,047) et au niveau de l'ensemble du site $(C \gamma=0,025)$. Pour ce qui concerne les caractéristiques de cisaillement $\left(c^{\prime}, \varphi^{\prime}\right)$, le faible nombre d'essais réalisés (16 essais de cisaillement sur les trois couches de sol) et l'hétérogénéité des formations testées ne permettent pas de porter un jugement significicatif. Des études récentes (Harr, 1977; Magnan, 1984...) montrent que le poids volumique présente toujours une faible dispersion autour de la moyenne alors que les paramètres de cisaillement ont une variabilité nettement plus élevée.

Les hypothèses suivantes ont donc été adoptées: - la géométrie, la pression interstitielle, le poids volumique et les caractéristiques du sol constituant les deux remblais ont été considérés comme des variables déterministes :

- la cohésion et l'angle de frottement effectifs des trois couches de sols sont les seules variables aléatoires.

Pour ce qui concerne le choix des lois de distribution de ces paramètres aléatoires, le faible nombre d'essais exécutés sur le site ne permet pas de définir précisément les lois de distributions. Divers auteurs (Lumb, 1966 et 1970; Schultze, 1972; Singh, 1972; Magnan et Baghery, 1980) ont montré que les paramètres de cisaillement suivent généralement une loi proche de la normale. La loi de distribution normale a donc été adoptée dans cette analyse.

La stratégie de recherche de la surface de rupture correspondant au risque maximal est analogue à celle des calculs classiques de stabilitẻ de pente: balayage de toutes les forces possibles, avec tous les jeux possibles de paramètres mécaniques. Ce type de calcul a été exécutê sur quelques ouvrages. Néanmoins, compte
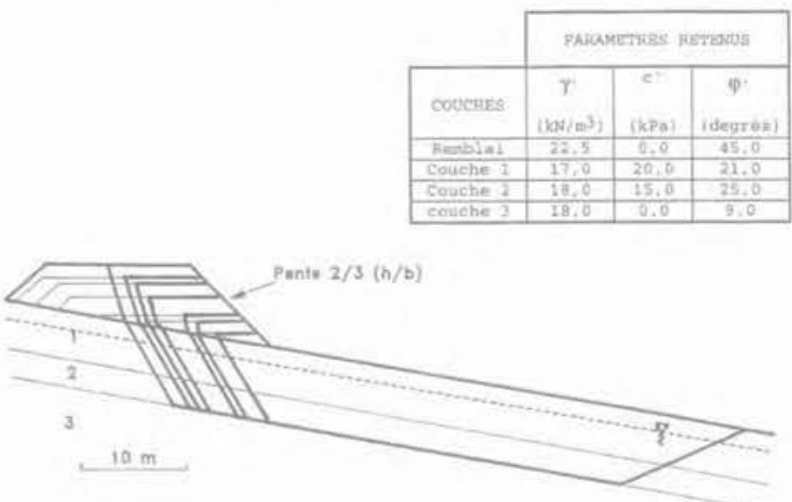

FG. 2 Remblai A de Sallèdes. Évolution des surfaces de rupture les plus défavorables lors de la construction du remblai. Salledes embankment A: evolution of the critical slip surface as construction of the embankment proceeds.

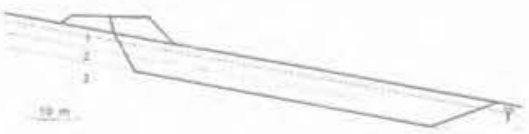

FIG. 3 Remblai B de Sallèdes. Calcul à la hauteur de $3,4 \mathrm{~m}$ : surface de rupture la plus défavorable.

Salledes embankment B: critical slip surface for an embankment height of $3.4 \mathrm{~m}$. 
tenu de la grande durée de ces calculs, une démarche simplifiée a été définie, après analyse des premiers résultats: on commence par rechercher la surface de rupture la plus défavorable en utilisant les valeurs moyennes de chaque paramètre aléatoire (calcul déterministe). Le calcul probabiliste est ensuite réalisé sur cette surface de rupture.

\section{2}

\section{Étude de la stabilité des deux remblais}

Les deux remblais ont été construits avec les mêmes caractéristiques géométriques à la base $(70 \times 13 \mathrm{~m})$. Les matériaux utilisés sont des graves alluvionnaires de l'Allier (granulométrie 0/60), qui ont été mises en œuvre par couches de $5 \mathrm{~cm}$ d'épaisseur environ.

Le remblai $\mathrm{A}$ (Fig. 2) a été monté à une hauteur de $5,4 \mathrm{~m}$ en 7 jours. L'analyse probabiliste de stabilité a été réalisée à la hauteur atteinte à la fin de chaque journée de travail ainsi qu'à la hauteur correspondant à la rupture observée (la schématisation de la surface du terrain naturel conduit à $\mathrm{H}_{\max }=5,10 \mathrm{~m}$ ).

Le remblai B (Fig. 3) a été monté à une hauteur de $3,4 \mathrm{~m}$ en deux phases (de 5 et 2 jours) espacées de 6 mois. L'analyse probabiliste de stabilité a été réalisée à la hauteur finale.

Trois couches de sol ont été prises en considération (colluvions, marnes remaniées et couche contenant la surface de glissement), avec les paramètres suivants:

\begin{tabular}{|c|c|c|c|}
\hline \multirow[b]{2}{*}{ COUCHES } & \multicolumn{3}{|c|}{ PARAME广TRES RETENUS } \\
\hline & $\begin{array}{c}\gamma \\
\left(\mathrm{kN} / \mathrm{m}^{3}\right)\end{array}$ & $\begin{array}{c}\mathrm{c}^{\prime} \\
(\mathrm{kPa})\end{array}$ & $\begin{array}{c}\varphi^{\prime} \\
\text { (degrés) }\end{array}$ \\
\hline $\begin{array}{l}\text { Remblai } \\
\text { Couche } 1 \\
\text { Couche } 2 \\
\text { Couche } 3\end{array}$ & $\begin{array}{l}22,5 \\
17,0 \\
18,0 \\
18,0\end{array}$ & $\begin{array}{c}0,0 \\
20,0 \\
15,0 \\
0,0\end{array}$ & $\begin{array}{c}45,0 \\
21,0 \\
25,0 \\
9,0\end{array}$ \\
\hline
\end{tabular}

La probabilité de rupture $\mathrm{P}$ est estimée en supposant que le coefficient de sécurité F suit une loi de distribution normale de moyenne $m_{\mathrm{F}}$ et d'écart type $\sigma_{\mathrm{F}}\left(\mathrm{m}_{\mathrm{F}}\right.$ et $\sigma_{F}$ sont calculés par la méthode d'intégration numérique): $\mathrm{P}$, est la probabilité que $\mathrm{F}<1$.

Le tableau I présente les résultats obtenus pour les différentes étapes de la construction du remblai $\mathrm{A}$.

Le tableau II présente les résultats obtenus pour chacun des remblais $A$ et $B$ à leur hauteur finale. Pour l'ensemble de ces calculs, les valeurs moyennes de la cohésion et de l'angle de frottement interne des deux premières couches de sols (Fig. 2 et 3 ) ont été fixées à:

$$
\text { Couche 1: } \begin{aligned}
c^{\prime} & =20 \mathrm{kPa} \\
\varphi^{\prime} & =21^{\circ} \\
\text { Couche } 2: c^{\prime} & =15 \mathrm{kPa} \\
\varphi^{\prime} & =25^{\circ}
\end{aligned}
$$

Les résultats sont présentés sous forme:

- de calculs déterministes sur la base d'une part du programme de calcul PETAL-LCPC (logiciel de calcul de stabilité mis au point par le Laboratoire Central des Ponts et Chaussées) et d'autre part de la méthode des blocs;

- de calculs probabilistes sur la base de la méthode d'intégration numérique.

La figure 4 illustre l'évolution de la probabilité de rupture en fonction de la variation de l'écart type $\sigma$. de l'angle de frottement interne de la troisième couche du sol pour une variation de 1 à 5 degrés de l'écart type de $\varphi^{\prime}$ et de 1 à $5 \mathrm{kPa}$ de l'écart type de $\mathrm{c}^{\prime}$ des deux autres couches du sol.

On constate une cohérence remarquable entre les résultats des calculs effectués par le logiciel PETALLCPC (calcul par la méthode des perturbations) et par la méthode des blocs, malgré les hypothèses géométriques simplificatrices effectuées pour ces derniers calculs.

La montée du remblai $\mathrm{A}$ entre les hauteurs $1,03 \mathrm{~m}$ et

\begin{tabular}{|c|c|c|c|c|c|c|c|c|}
\hline & \multirow{2}{*}{\multicolumn{2}{|c|}{$\begin{array}{l}\text { Calcul } \\
\text { déterministe }\end{array}$}} & \multicolumn{6}{|c|}{ Calcul probabiliste (blocs) } \\
\hline & & & \multicolumn{6}{|c|}{ Valeurs moyennes comme pour le calcul déterministe } \\
\hline $\begin{array}{c}\text { Paramètres } \\
\text { de } \\
\text { calcul }\end{array}$ & \multicolumn{2}{|c|}{$\begin{array}{l}\text { Couche } 1 \\
\mathrm{~m}_{e^{\prime}}=20 \mathrm{kPa} \mathrm{m}_{\psi}=21 \text { degrés } \\
\text { Couche } 2 \\
\mathrm{~m}_{c^{\prime}}=15 \mathrm{kPa} \mathrm{m}_{\psi^{\prime}}=25 \text { degrés } \\
\text { Couche } 3 \\
\mathrm{~m}_{c^{\prime}}=0 \quad \mathrm{~m}_{\sigma^{\prime}}=9 \text { degrés }\end{array}$} & \multicolumn{3}{|c|}{$\begin{array}{ll}\text { Couches } 1 \text { et } 2 & \\
\sigma_{c}=5 \mathrm{kPa} & \sigma_{*}=4 \text { degrés } \\
\text { Couche } 3 & \\
\sigma_{c}=0 & \sigma_{e}=4 \text { degrés }\end{array}$} & \multicolumn{3}{|c|}{$\begin{array}{ll}\text { Couches } 1 \text { et } 2 \\
\begin{array}{ll}\sigma_{c^{\prime}}=3 \mathrm{kPa} & \sigma_{\varphi^{\prime}}=3 \text { degrés } \\
\text { Couche } 3 & \\
\sigma_{\mathrm{c}^{\prime}}=0 & \sigma_{\varphi^{\prime}}=2 \text { degrés }\end{array}\end{array}$} \\
\hline $\begin{array}{l}\text { Hauteur } \\
\text { (m) }\end{array}$ & $\begin{array}{c}\text { PETAL-LCPC } \\
\text { F }\end{array}$ & $\begin{array}{c}\text { Blocs } \\
\text { F }\end{array}$ & $\mathrm{m}_{\mathrm{F}}$ & $\sigma_{\mathrm{F}}$ & $P_{f}$ & $\begin{array}{l}\mathrm{m}_{\mathrm{F}} \\
(\%)\end{array}$ & $\sigma_{\bar{F}}$ & $\begin{array}{l}\mathrm{P}_{r} \\
(\%)\end{array}$ \\
\hline $\begin{array}{l}1,03 \\
1,74 \\
2,06 \\
3,09 \\
3,80 \\
4,36 \\
5,10\end{array}$ & $\begin{array}{l}1,593 \\
1,378 \\
1,318 \\
1,243 \\
1,134 \\
1,077 \\
1,021\end{array}$ & $\begin{array}{l}1,430 \\
1,301 \\
1,251 \\
1,127 \\
1,055 \\
1,020 \\
0,973\end{array}$ & $\begin{array}{l}1,436 \\
1,306 \\
1,256 \\
1,130 \\
1,058 \\
1,023 \\
0,975\end{array}$ & $\begin{array}{l}0,248 \\
0,222 \\
0,212 \\
0,190 \\
0,177 \\
0,171 \\
0,163\end{array}$ & $\begin{array}{r}3,62 \\
8,38 \\
11,31 \\
24,83 \\
37,07 \\
44,83 \\
55,96\end{array}$ & $\begin{array}{l}1,432 \\
1,303 \\
1,253 \\
1,128 \\
1,056 \\
1,021 \\
0,74\end{array}$ & $\begin{array}{l}0,133 \\
0,119 \\
0,114 \\
0,102 \\
0,095 \\
0,974 \\
0,088\end{array}$ & $\begin{array}{r}0,057 \\
0,56 \\
1,33 \\
10,40 \\
27,80 \\
40,90 \\
61,50\end{array}$ \\
\hline
\end{tabular}
$5,10 \mathrm{~m}$ conduit à une diminution sensible du coefficient

tabieANI Calculs de stabilité du remblai A de Sallèdes au cours de la construction. Stability analyses of Salledes embankment A during its constuction. 


\begin{tabular}{|c|c|c|c|c|c|c|c|c|c|}
\hline & & \multirow{2}{*}{\multicolumn{2}{|c|}{$\begin{array}{c}\text { Calcul } \\
\text { déterministe }\end{array}$}} & \multicolumn{6}{|c|}{ Calcul probabiliste (blocs) } \\
\hline & & & & \multicolumn{6}{|c|}{ Valeurs moyennes comme pour le calcul déterministe } \\
\hline \multicolumn{2}{|c|}{$\begin{array}{c}\text { Paramètres } \\
\text { de } \\
\text { calcul }\end{array}$} & \multicolumn{2}{|c|}{$\begin{array}{l}\text { Couche } 1 \\
\mathrm{~m}_{c}=20 \mathrm{kPa} \mathrm{m}_{\psi^{\prime}}=21 \text { degrés } \\
\text { Couche } 2 \\
\mathrm{~m}_{c}=15 \mathrm{kPa} \mathrm{m}_{\psi^{*}}=25 \text { degrés } \\
\text { Couche } 3 \\
\mathrm{~m}_{\tau^{\prime}}=0 \quad \mathrm{~m}_{\psi}=9 \text { degrés }\end{array}$} & \multicolumn{3}{|c|}{$\begin{array}{ll}\text { Couches } 1 \text { et } 2 \\
\sigma_{c^{\prime}}=2 \mathrm{kPa} & \sigma_{\varphi^{\prime}}=3 \text { degrés } \\
\text { Couche } 3 & \\
\sigma_{c^{\prime}}=0 & \sigma_{4^{\prime}}=3 \text { degrés }\end{array}$} & \multicolumn{3}{|c|}{$\begin{array}{ll}\text { Couches } 1 \text { et } 2 \\
\sigma_{c^{\prime}}=2,5 \mathrm{kPa} & \sigma_{\varphi^{\prime}}=2 \text { degrés } \\
\text { Couche } 3 & \\
\sigma_{c^{\prime}}=0 & \sigma_{\varphi^{\prime}}=1 \text { degré }\end{array}$} \\
\hline \multicolumn{2}{|c|}{ Couche 3} & PETAL-LCPC & Blocs & $\mathrm{m}_{\mathrm{F}}$ & $\sigma_{F}$ & $\mathrm{P}_{r}$ & $m_{F}$ & $\sigma_{F}$ & $\mathrm{P}_{\mathrm{r}}$ \\
\hline $\mathrm{m}_{c}$ & $\mathrm{~m}_{6}$ & F & $\mathrm{F}$ & & & $(\%)$ & & & $(\%)$ \\
\hline $\begin{array}{r}0 \\
0 \\
10 \\
10\end{array}$ & $\begin{array}{r}9 \\
18 \\
9 \\
18\end{array}$ & $\begin{array}{l}1,021 \\
1,432 \\
1,449 \\
1,711\end{array}$ & $\begin{array}{l}0,973 \\
1,294 \\
1,289 \\
1,597\end{array}$ & $\begin{array}{l}0,975 \\
1,296 \\
1,290 \\
1,598\end{array}$ & $\begin{array}{l}0,116 \\
0,113 \\
0,111 \\
0,113\end{array}$ & $\begin{array}{c}58,70 \\
0,45 \\
0,44 \\
0,53 \times 10^{-5}\end{array}$ & $\begin{array}{l}0,974 \\
1,294 \\
1,290 \\
1,597\end{array}$ & $\begin{array}{l}0,051 \\
0,051 \\
0,051 \\
0,051\end{array}$ & $\begin{array}{c}69,60 \\
0,50 \times 10^{-6} \\
0,32 \times 10^{-6} \\
0,38 \times 10^{-29}\end{array}$ \\
\hline
\end{tabular}

b. Remblai $\mathrm{B}(\mathrm{H}=3,4 \mathrm{~m})$

b. Embankment B (height: $3.4 \mathrm{~m}$ )

\begin{tabular}{r|r|c|c|c|c|c|c|c|c}
\hline \multicolumn{2}{c|}{ Couche 3 } & PETAL-LCPC & Blocs & $\mathrm{m}_{\mathrm{F}}$ & $\sigma_{\mathrm{F}}$ & $\mathrm{P}_{\mathrm{r}}$ & $\mathrm{m}_{\mathrm{F}}$ & $\sigma_{\mathrm{F}}$ & $\mathrm{P}_{\mathrm{r}}$ \\
\hline $\mathrm{m}_{\tau}$ & $\mathrm{m}_{\mathrm{\sigma}}$ & $\mathrm{F}$ & $\mathrm{F}$ & & & $(\%)$ & & & $(\%)$ \\
\hline 0 & 9 & 0,985 & 1,041 & 1,043 & 0,146 & 38,40 & 1,041 & 0,059 & 24,30 \\
0 & 18 & 1,478 & 1,464 & 1,467 & 0,151 & 0,098 & 1,465 & 0,061 & $0,15 \times 10^{-11}$ \\
10 & 9 & 1,476 & 1,493 & 1,495 & 0,143 & 0,027 & 1,494 & 0,059 & $0,18 \times 10^{-14}$ \\
10 & 18 & 1,952 & 1,907 & 1,910 & 0,149 & $0,49 \times 10^{-7}$ & 1,907 & 0,061 & $0,94 \times 10^{-4}$ \\
\hline
\end{tabular}

de sécurité de 1,43 à 0,97 et à une augmentation de la probabilité de rupture de 0,057 à $61,5 \%$.

Le choix des caractéristiques de cisaillement de la troisième couche $\left(\varphi^{\prime}=9\right.$ à $\left.18^{\circ}\right)$ a une grande influence, tant sur la valeur du coefficient de sécurité que sur celle de la probabilité de rupture.

On peut voir qu'une augmentation de 1 à $5^{\circ}$ de l'écart type de l'angle de frottement interne de la couche 3 (cas où $\varphi^{\prime}=18^{\circ}$ ) conduit à des variations très importantes de la probabilité de rupture (de $36.10^{-19}$ à $3 \%$ ) en considérant des valeurs constantes pour les paramètres des couches 1 et 2. Dans le cas d'une augmentation de 1 à 5 de l'écart type des paramètres des couches 1 et 2, en maintenant constantes les caractéristiques de la couche 3 , on observe une augmentation nettement moindre de la probabilité de rupture $(0,08$ à 0,26$)$.

On remarque donc que la variation de l'écart type de l'angle de frottement interne $\varphi$ ' mesuré dans la troi- sième couche (couche où se produit l'essentiel du glissement) a une influence sur la probabilité de rupture plus importante que celle produite par la variation des écarts types des paramètres mesurés dans les autres couches.

La grande sensibilité de la probabilité de rupture aux variations des paramètres statistiques caractérisant les propriétés des sols montre que l'application des méthodes de calcul probabilistes (qui tiennent compte de la variabilité des propriétés des sols) nécessite une évaluation soigneuse des paramètres statistiques des propriétés considérées comme variables aléatoires dans le calcul. Cette évaluation doit être effectuée en tenant compte des variations spatiales des paramètres considérés, ce qui produit habituellement des valeurs faibles des écarts types, de l'ordre de celles qui ont été utilisées dans les études paramétriques de la figure 4. 


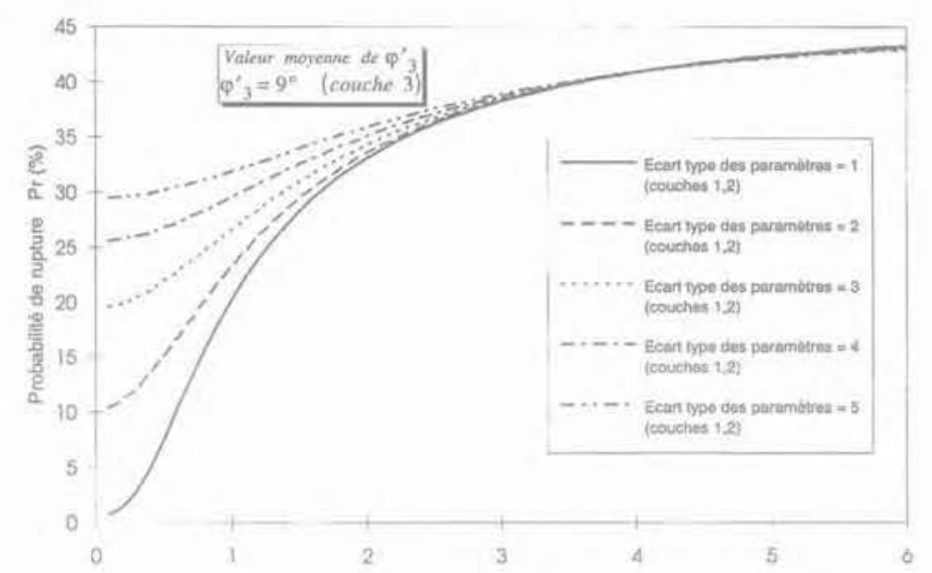

Écart-type de l'angle de frottement interne de la couche $3 \sigma \varphi_{3}^{\prime}$ (degrés)

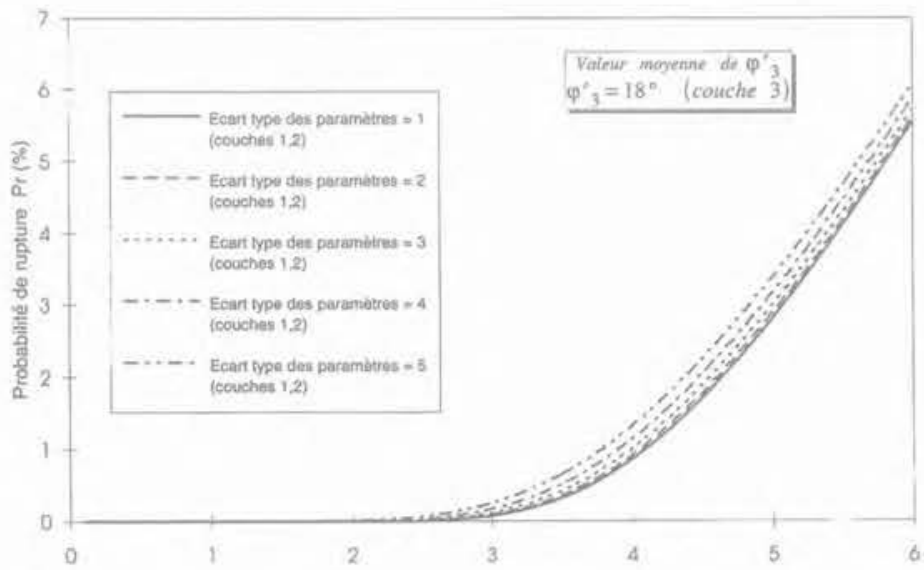

Écart-type de l'angle de frottement interne de la couche $3 \sigma \varphi_{3}^{\prime}$ (degrés)

FiG.4 Variations de la probabilité de rupture du remblai B en fonction des écarts types des paramètres des sols (site de Sallèdes).

Probability of failure of embankment B versus standard deviations of the soil characteristics (Salledes experimental site).

\section{6}

\section{Conclusion}

L'analyse probabiliste de la stabilité des deux remblais construits sur le versant instable de Sallèdes présentée dans cet article a consisté à évaluer la probabilité de rupture de ces remblais sur la base de la méthode des blocs et d'une procédure d'intégration numérique.

Les paramètres de cisaillement considérés comme variables aléatoires dans cette étude sont la cohésion effective et l'angle de frottement interne effectif (étude de stabilité d'ensemble à long terme). La loi de distri- bution normale a été choisie pour représenter la répartition des valeurs de ces deux paramètres.

L'introduction des méthodes probabilistes dans le calcul de stabilité a eu un effet non négligeable sur l'estimation du niveau de risque, qui est tout à fait conforme aux variations qualitatives que l'on peut déduire d'un raisonnement empirique.

Si l'on exclut les incertitudes résultant de la méthode d'intégration numérique, et par comparaison avec les résultats des calculs déterministes, on peut donc dire que l'analyse statistique et probabiliste effectuée a donné des résultats raisonnables et encourageants, dont l'utilité pour les études de projets courants doit maintenant être étudiée, à la suite de cette première recherche. 
Abdul Baki A. - Analyse probabiliste du comportement de deux remblais sur versant instable. Thèse de doctorat, Institut National des Sciences Appliquées de Lyon, 1992, $290 \mathrm{p}$.

Blondeau F., Morin P., Pouget P. - Comportement d'un remblai construit jusqu'â la rupture sur un versant naturel, site expérimental de Sallèdes (Puyde-Dóme). Paris, Laboratoire Central des Ponts et Chaussées, Rapport de recherche LPC, $\mathrm{n}^{\circ} 126,1983,46 \mathrm{p}$.

Cartier G. Pouget P. - Étude du comportement d'un remblai construit sur versant instable, le remblai de Sallèdes (Puy-deDòme). Paris, Laboratoire Central des Ponts et Chaussées, Rapport de Recherche LPC, $n^{\circ} 153,1988,130 \mathrm{p}$.

Evans D.H. - The application of numerical integration techniques to statistical tolerancing. Technometrics, vol. $9 . n^{\circ} 3$, 1967, pp. 441-456.

Evans D.H. - An application of numerical integration techniques to statistical tole- rancing. III General distributions, Technometrics, vol. $14, n^{\circ} 1,1972, p p .23-35$.

Harr M.E. - Mechanics of particulate media. A probabilistic approach, 1977 . New York, Mac Graw-Hill.

Lumb P. - Variability of natural soils. Canadian Geotechnical Journal, vol. $3, n^{\circ} 2$. 1976, pp. 74-97.

Lumb P. - Safety factors and the probability distribution of soil strength. Canadian Geotechnical Journal, vol. 7, n⿳3 1970, pp, 225-242

Lumb P - Application of statistics in soil mechanics. In "Soil Mechanics: New Horizons v, 1974, New York. American Elsevier Publ. Company, Inc

Magnan J.-P. - Les méthódes statistiques et probabilistes en mécanique des sols Presses de l'Ecole Nationale des Ponts et Chaussées, 1982, Paris, $203 \mathrm{p}$.

Magnan J.-P. - Méthodes statistiques en mécanique des sols. Comptes rendus. Journées de Mécanique aléatoire appliquée à la construction. Paris. Juin 1984.
Association Francaise de Recherche et d'Essais sur les Matériaux et les Constructions (AFREM), pp. 217-225.

Magnan J.-P. Baghery S. - Etude probabiliste du comportement d'un remblai sur sols mous. Revue Française de Géotechnique, $\mathrm{n}^{\circ} 20,1982$, pp. 5-11.

Morin P. - Ettude du comportement avant la rupture d'un remblai expérimental sur versant à Sallèdes (Puy-de-Dóme). Thèse de docteur ingénieur, Ecole Nationale des Ponts et Chaussèes, Paris, 1979, 318p.

Schultze E. - Frequency distributions and correlation of soil properties, Proceedings, 1st International Conference or Applications of Statistics and Probability in Soil and Structural Engineering, Hong-Kong, 1972, pp. 371-388.

Singh A. - How reliable is the factor of safety in foundation engineering? Proceedings, 1st international Conference on Applications of Statistics and Probability in Soil and Structural Engineering Hong Kong, 1972, pp. 389-424.

\section{$13^{e}$ Réunion générale annuelle de l'Association Canadienne des Travaux en Souterrains Appel à communication}

L'Association Canadienne des Travaux en Souterrains tiendra sa $13^{e}$ Conférence sur les tunnels ainsi que sa réunion annuelle à Montréal, Province du Québec (Canada),

\section{du 18 au 20 octobre 1995}

\section{- Thème de la Conférence}

Le but de la Conférence de 1995 est de présenter l'état de l'art dans le domaine de la construction des tunnels. Elle rassemblera des présentations sur des développements récemment terminés ou en cours, aussi bien que sur des exemples de pratique courante ou sur des études de cas, Les aspects d'ingénierie seront privilégiés. Des contributions traitant de l'emploi des matériels sont particulièrement souhaitées:

\section{- Appel à communication}

Les auteurs sont invités à envoyer un résumé de moins de 500 mots au Comité du Programme Technique de la Conférence (CPTC) à l'adresse ci-dessous. Les communications devront ètre originales et en accord avec le thème de la Conférence.

- Date limite de réception des résumés 28 février 1995

- Notification aux auteurs 31 mars 1995

- Date limite de réception des communications : 15 juin 1955

sur des formes prètes à la reprographie

La sélection des communications est sous la responsabilité du CPTC. Les résumés seront revus par au moins deux lecteurs.

\section{- Comptes rendus}

Les communications acceptées par la CPTC seront publiées dans un volume qui sera distribué à chaque participant.

En plus, toutes les communications acceptées seront revues par le Bureau éditorial du Canadian Tunnelling Journal et les textes acceptés seront aussi publiés par le Journal.

Adresse: Denis E. Gill

Tél. : (514) 340-5951

TPC/1995 TAC Conference

Fax: (514) 340-4477

Ecole Polytechnique

CP 6079, Succursale Centre-Ville

MONTREAL, QC, Canada

$\mathrm{H} 3 \mathrm{C} 3 \mathrm{~A} 7$ 